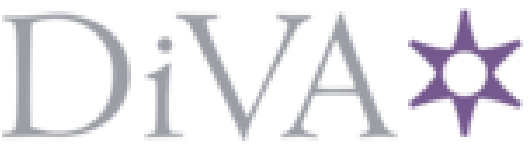

http://www.diva-portal.org

\title{
Postprint
}

This is the accepted version of a paper presented at CAMA 2017, December 4-6, Tsukuba, Japan.

Citation for the original published paper:

Asan, N B., Velander, J., Redzwan, S., Augustine, R., Hassan, E. et al. (2017)

Reliability of the fat tissue channel for intra-body microwave communication.

In: Proc. 4th International Conference on Antenna Measurements and Applications IEEE

N.B. When citing this work, cite the original published paper.

Permanent link to this version:

http://urn.kb.se/resolve?urn=urn:nbn:se:uu:diva-335483 


\section{Reliability of the Fat Tissue Channel for Intra-body Microwave Communication}

\author{
${ }^{1,2}$ Noor Badariah Asan, ${ }^{1}$ Jacob Velander, ${ }^{1}$ Syaiful \\ Redzwan, ${ }^{1}$ Robin Augustine \\ ${ }^{1}$ Microwave group, Department of Engineering Sciences, \\ Solid State Electronics, Uppsala University, Sweden \\ ${ }^{2}$ Faculty of Electronic and Computer Engineering, \\ Universiti Teknikal Malaysia Melaka, Melaka, Malaysia. \\ noorbadariah.asan@angstrom.uu.se
}

\author{
Thiemo Voigt \\ Department of Information Technology, \\ Division of Computer Systems, \\ Uppsala University, Uppsala, Sweden.
}

\author{
${ }^{3,4}$ Emadeldeen Hassan, ${ }^{3}$ Daniel Noreland \\ ${ }^{3}$ Department of Computing Science, \\ Umeå University, Umeå, Sweden \\ ${ }^{4}$ Department of Electronics and Electrical Communications, \\ Menoufia University, \\ Menouf, Egypt.
}

\begin{abstract}
Recently, the human fat tissue has been proposed as a microwave channel for intra-body sensor applications. In this work, we assess how disturbances can prevent reliable microwave propagation through the fat channel. Perturbants of different sizes are considered. The simulation and experimental results show that efficient communication through the fat channel is possible even in the presence of perturbants such as embedded muscle layers and blood vessels. We show that the communication channel is not affected by perturbants that are smaller than $15 \mathrm{~mm}$ cube.
\end{abstract}

Keywords- microwaves; dielectric properties; fat channel; intra-body communication; phantom.

\section{INTRODUCTION}

Over the past few years, intra-body communication has attracted increasing attention and has been considered for applications such as biomedical sensor networks [1], manmachine interfaces [2], and drug delivery systems [3]. The most commonly used methods for the intra-body communication for body-area networks are galvanic and capacitive coupling. The authors in [4] reviewed and compared both methods in term of their performance and limitations.

We proposed the new method of communication through fat (adipose) in [5] and [6], where the fat tissue was used as a channel for microwave propagation through the human body. The new method offers higher data rates and integrity. In [7], we have also shown that the fat channel can be used to transmit and receive data packets. Along with fat cells, adipose tissue contains numerous nerve cells and blood vessels [8]. Therefore, it is important to consider disturbances caused by embedded muscles and blood vessels in the fat channel to assess the reliability of intra-body microwave communication. The signal coupling is considered as an important parameter to assess the channel quality. To the best of our knowledge, there is no published analysis about the effect of such perturbants on the fat tissue communication channel.

\author{
Taco J. Blokhuis \\ Department of Surgery, \\ Maastricht University Medical Center+, \\ Maastricht, The Netherland.
}

\section{MATERIALS AND METHODS}

\section{A. Numerical Modeling}

For the numerical modeling, we consider a homogeneous three-layer model consisting of skin, fat, and muscle tissue. The dielectric properties of tissues equivalent phantoms were imported to the Computer Simulation Technology (CST) microwave studio - 3D Electromagnetic (EM) simulation software [9]. Fig. 1 shows the symmetrical view of the simulation setup. The thicknesses of the skin, fat, and muscle tissue in this study are selected to be $2 \mathrm{~mm}, 25 \mathrm{~mm}$, and $30 \mathrm{~mm}$ thick, respectively as in [5]. The $25 \mathrm{~mm}$ thick fat layer sandwiched between the skin and muscle layer formed the fat channel. Probe 1, as shown in Fig. 1, transmits the microwave signal through the $100 \mathrm{~mm}$ long fat channel and probe 2 acts as receiver. First, we simulate the reference channel without any perturbation to observe the effect of any disturbance occurring during the signal transmission. In real-world scenarios, the fat tissue layer might contain embedded muscles or blood vessels. To mimic this case, a perturbant tissue (PT) made of muscle phantom embedded in the center of the fat channel is considered. The muscle phantom was chosen to be a perturbant because it has high dielectric properties compared to fat that may change the behavior of the channel. The blood vessels also have similar dielectric properties as muscle tissues [10]. Therefore, different PT sizes of $5 \mathrm{~mm}, 10 \mathrm{~mm}, 15 \mathrm{~mm}$, and 20 $\mathrm{mm}$ cubes are used in the simulation.

The PT location inside the fat channel is moved laterally from probe 1 to probe 2 in $20 \mathrm{~mm}$ steps. This helps us to assess the effect of the perturbant position on signal coupling through the fat channel. The skin and muscle tissue are made from agar-based phantom material, and the fat tissue is a rubber block with dielectric properties equivalent to the tissue. The probes used in this work are topology optimized planar antenna (TOPA) based waveguide transition [11]. TOPA waveguide 
transitions are optimized for the R-band frequency range to launch the electromagnetic waves into the fat channel.

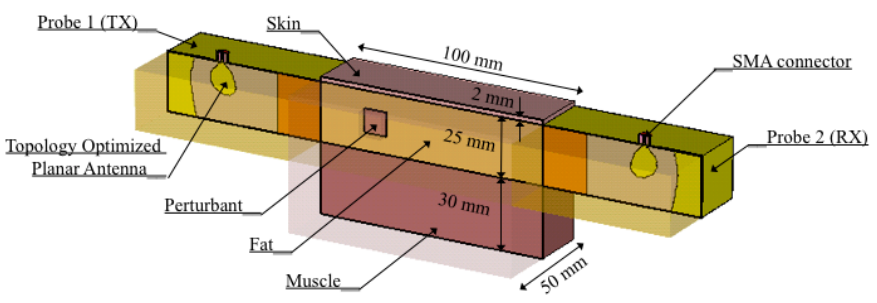

Fig. 1. Simulation setup on CST software (symmetrical)

Fig. 2 and Fig. 3 shows the E-field at different crosssections of the simulation setup with probe 1, channel embedded with the PT, and probe 2 . The simulation was done with a PT size equal to $10 \mathrm{~mm}$ and $15 \mathrm{~mm}$. The E-field is simulated at $2.0 \mathrm{GHz}$ when the PT is placed at $40 \mathrm{~mm}$ distance from the probe 1 (transmitter). Fig. 2 and Fig. 3 (a), (b), (c), and $(\mathrm{d})$ represent the $\mathrm{Y}-\mathrm{Z}$ cross-sectional E-field distribution at the center of the phantom, the $X-Z$ cross-sectional E-field distribution along the channel, X-Y cross-sectional E-field distribution along the channel, and the scalar color mapping, respectively. Fig. 2 and Fig. 3 show the simulation results with a PT size of $10 \mathrm{~mm}$, and $15 \mathrm{~mm}$, respectively.

Fig. 2 and Fig. 3 show that the E-field is propagating from probe 1 to probe 2 through the fat layer. It can also see that the PT is situated in the propagation path representing fat channel perturbation due to embedded muscle or blood vessels. Since the skin and the muscle layer have higher electrical conductivity than the fat layer, they confine the electrical field forming a channel through the fat tissue layer. The PT offers minimal obstruction to the E-field distribution in both cases as shown in Fig. 2 and Fig. 3.

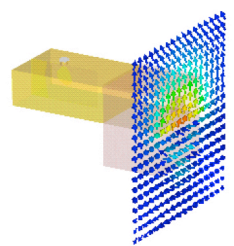

(a)

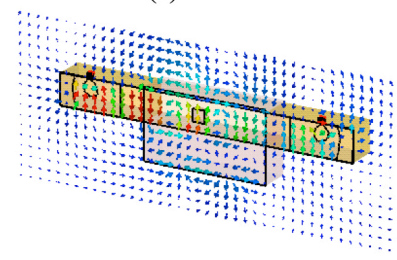

(c)

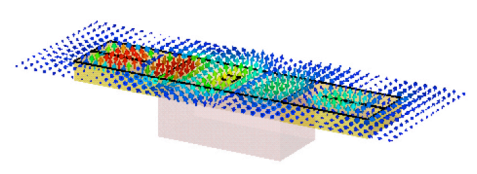

(b)

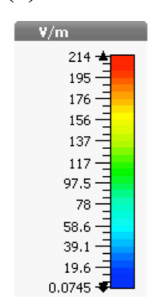

(d)
Fig. 2. E-field at $2.0 \mathrm{GHz}$ for $10 \mathrm{~mm}$ perturbant tissue size at $40 \mathrm{~mm}$ distance (a) cross-section Y-Z (b) cross-section X-Z, (c) cross-section X-Y, (d) scalar color mapping

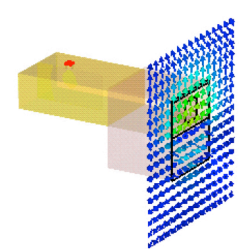

(a)

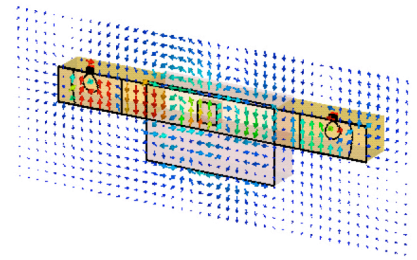

(c)

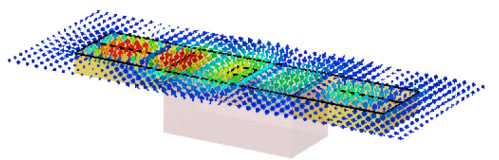

(b)

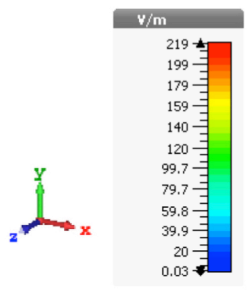

(d)
Fig. 3. E-field at $2.0 \mathrm{GHz}$ for $15 \mathrm{~mm}$ perturbant tissue size at $40 \mathrm{~mm}$ distance (a) cross-section $\mathrm{Y}-\mathrm{Z}$ (b) cross-section $\mathrm{X}-\mathrm{Z}$, (c) cross-section $\mathrm{X}-\mathrm{Y}$, (d) scalar color mapping

\section{B. Phantom measurement setup}

To validate the results obtained through simulations performed with the CST model, we carry out the experimental studies on equivalent phantom tissue. From our previous studies [5], we see that the channel performs well around 2.0 $\mathrm{GHz}$. As shown in Fig. 4, the probe is resonant at $2.0 \mathrm{GHz}$ and at this frequency the correlation between the simulation and the measurement results is high.

Fig. 5 (a) shows the phantom experimental setup for the PT measurement. An enlarged image in Fig. 5 (b) shows the position of the PT inside the fat-equivalent phantom. The overall dimensions of the channel are $50 \mathrm{~mm}$ (width) $\times 100$ $\mathrm{mm}$ (length) $\times 67 \mathrm{~mm}$ (height). The probes are aligned with the fat layer of the phantom. The PT dimensions of $10 \mathrm{~mm} \times 10$ $\mathrm{mm} \times 10 \mathrm{~mm}$ and $15 \mathrm{~mm} \times 15 \mathrm{~mm} \times 15 \mathrm{~mm}$ cubical shape have been used in the experiment. The effect of the PT is assessed by measuring the scattering parameter (S-parameter) at every $20 \mathrm{~mm}$ using a FieldFox microwave analyzer (N9918A) by Agilent Technologies [12]. The skin, fat, and muscle tissue thicknesses were kept constant at $2 \mathrm{~mm}, 25 \mathrm{~mm}$, and $30 \mathrm{~mm}$, respectively for both measurements. The influence of the different PT size on the signal coupling was assessed by considering the variation in the amplitude of the transmission coefficient $\left(\mathrm{S}_{21}\right)$. We also compared the results with the fat channel, which is without any perturbants.

The dielectric properties of the phantom tissue samples were determined by an Agilent dielectric slim form probe (85070E) [13]. For each sample, the measurements were repeated 5 times at 801 discrete frequencies, evenly distributed in the logarithmic scale, over the frequency band $1-10 \mathrm{GHz}$. The results at each frequency were averaged. Table 1 shows the comparison of the relative permittivity and loss tangent between tissue-equivalent phantom and the human tissue at 2 $\mathrm{GHz}$. 


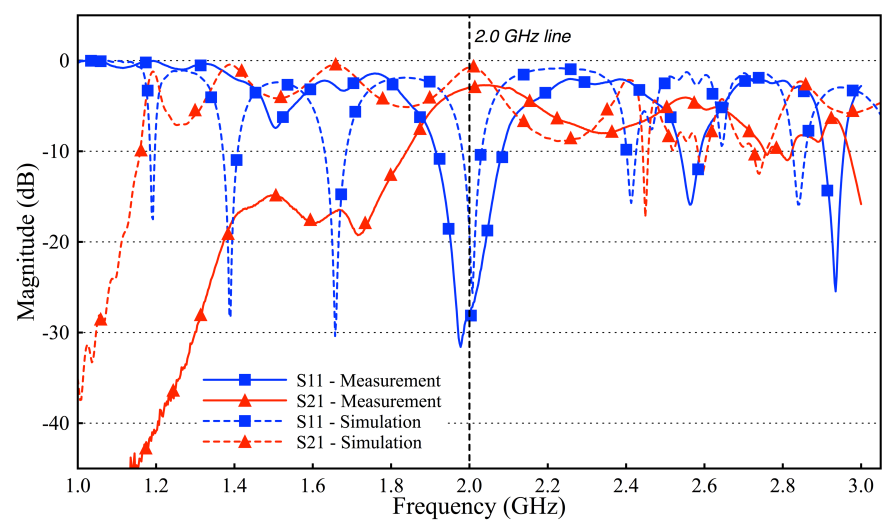

Fig. 4. Probe-to-probe: S-parameter simulation and measurement results

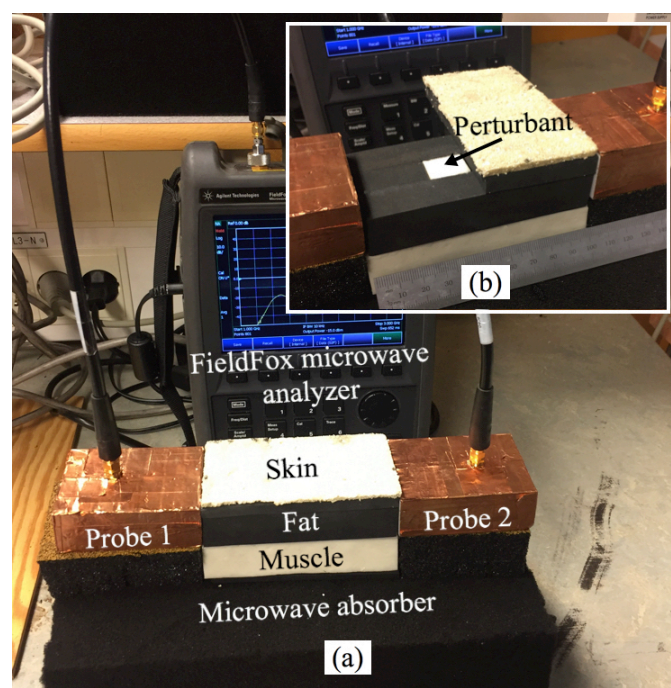

Fig. 5. Phantom experimental setup (a) full setup, (b) the perturbant inside fat tissue channel

TABLE I. COMPARISON OF THE DIELECTRIC PROPERTIES OF PHANTOM AND HUMAN TISSUES AT $2.0 \mathrm{GHz}[10]$

\begin{tabular}{|c|c|c|c|c|}
\hline \multirow{2}{*}{ Materials } & \multicolumn{4}{|c|}{ Parameters } \\
\cline { 2 - 5 } & \multicolumn{2}{|c|}{ Relative Permittivity } & \multicolumn{2}{c|}{ Loss tangent. tan $\boldsymbol{\delta}$} \\
\cline { 2 - 5 } & Phantom & Human & Phantom & Human \\
\hline Skin & 36.496 & 38.568 & 0.220 & 0.295 \\
\hline Fat & 5.433 & 5.328 & 0.156 & 0.145 \\
\hline Muscle & 53.860 & 53.290 & 0.207 & 0.245 \\
\hline
\end{tabular}

\section{RESULTS AND DISCUSSION}

\section{A. Simulation Results}

Fig. 6 shows the $\mathrm{S}_{21}$ simulation results with different PT sizes inside the fat channel. The solid line shows the reference, which is the fat channel without any disturbances. As shown in Fig. 6, there is almost no change in the amplitude of $S_{21}$ when the channel is perturbed with a PT of size $5 \mathrm{~mm}$ or $10 \mathrm{~mm}$ at any position. However, the signal coupling between the two probes starts to degrade when the channel is perturbated with a PT of size $15 \mathrm{~mm}$. As shown, the amplitude gradually decreases as the PT size increases to $20 \mathrm{~mm}$. The total thickness of the fat channel is $25 \mathrm{~mm}$, which means that only perturbants of $60 \%$ of the channel height or more will affect the channel performance. Also for other position of the perturbants, we notice similar results. We emphasize that the goal of the current work is to assess the reliability of the fat tissue as a communication channel. Therefore, we focus in presenting the $\mathrm{S}_{21}$ results.

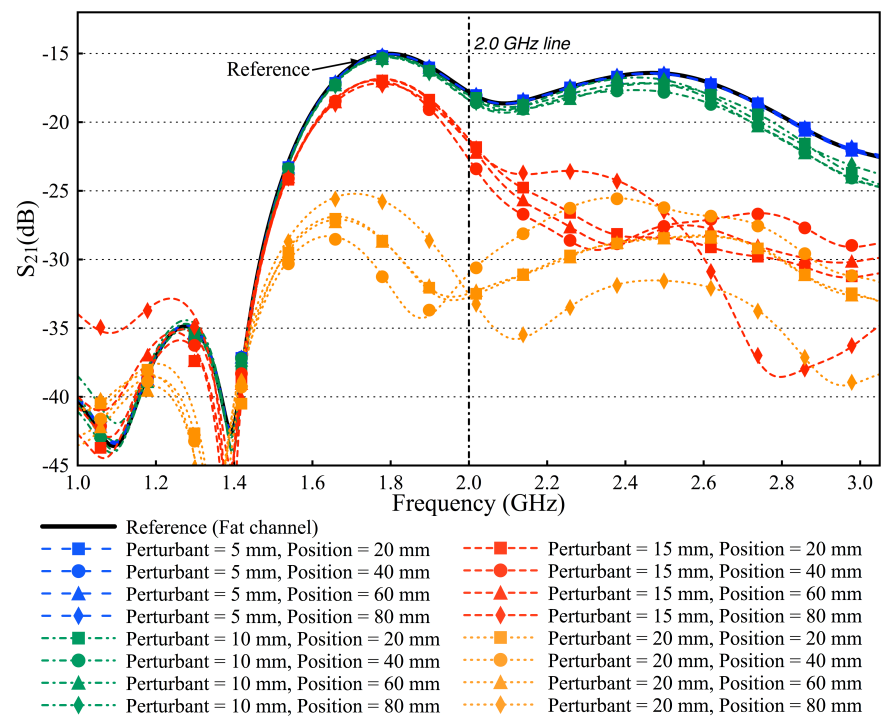

Fig. 6. Transmission coefficient, $S_{21}$ simulated results with different perturbant sizes at different position

\section{B. Measurement Results}

The S-parameter results for $10 \mathrm{~mm}$ PT size are depicted in Fig. 7. The solid black line with the square symbol is the reference line for the fat channel without any disturbances. The signal coupling does not change significantly by changing the position of the perturbant. We may conclude that the presence of the $10 \mathrm{~mm}$ PT does not strongly affect the microwave propagation through the fat channel.

The effect of PT on the fat channel is further analyzed using the $15 \mathrm{~mm}$ PT tissue. Fig. 8 shows the S-parameter comparison between the simulation and the measurement. It can be seen that the $S_{21}$ amplitude is decreased around $4 \mathrm{~dB}$ in the presence of $15 \mathrm{~mm}$ PT. The experimental results are in agreement with the simulated results as previously discussed in the Section III $(A)$. Fig. 6 also shows similar trends.

Fig. 9 presents the comparison between simulation and measurement of the PT sizes, $10 \mathrm{~mm}$ and $15 \mathrm{~mm}$ situated at 40 $\mathrm{mm}$ in the fat channel. The dashed line represents the simulation results while solid lines represent the measurement results. Both the simulation and the measurements show that a $10 \mathrm{~mm}$ PT does not affected the $\mathrm{S}_{21}$ but a $15 \mathrm{~mm}$ PT decreases the $S_{21}$ amplitude considerably. The shift in the frequency response and the difference in the amplitude in simulation and measurement can be accounted for differences in the dielectric properties between the simulated model and the phantom. 


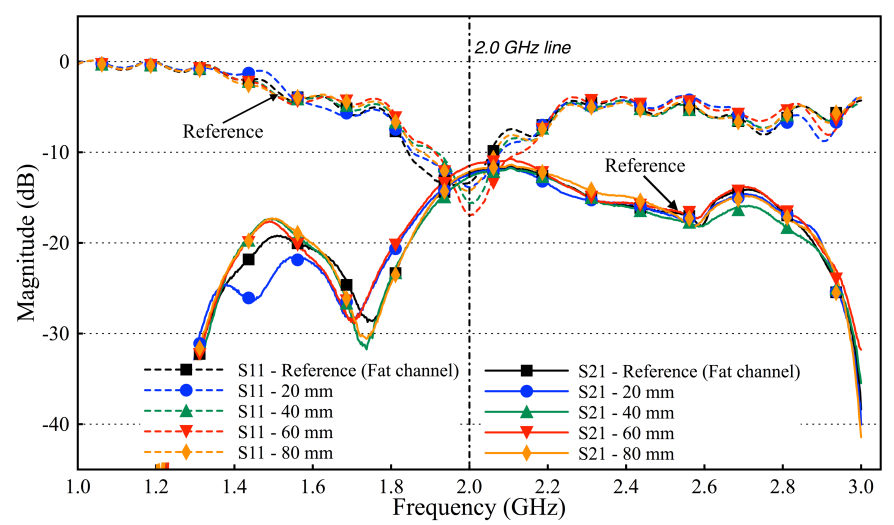

Fig. 7. S-parameters of $10 \mathrm{~mm}$ PT block (measurement)

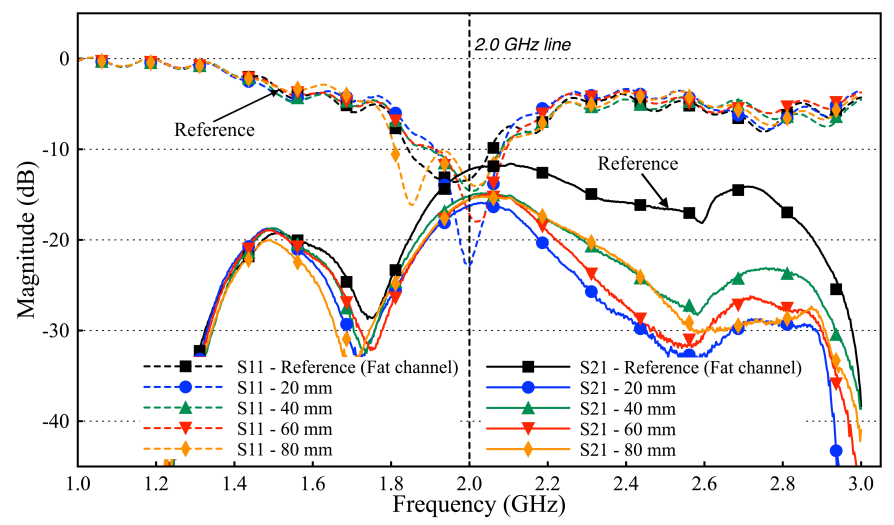

Fig. 8. S-parameters of $15 \mathrm{~mm}$ PT block (measurement)

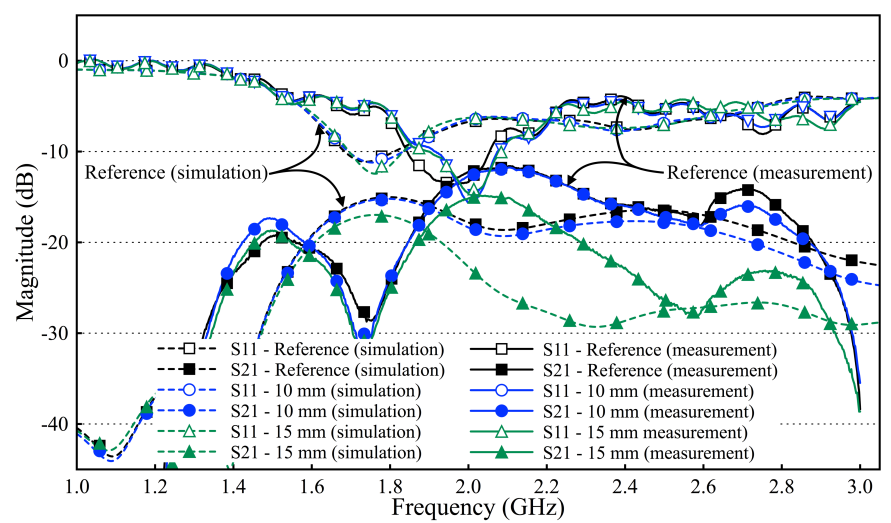

Fig. 9. Comparison between simulation and measurement results at $40 \mathrm{~mm}$ PT position for $10 \mathrm{~mm}$ and $15 \mathrm{~mm}$ PT sizes with respect to the reference (fat channel)

\section{CONCLUSIONS}

In this work, we have investigated the reliability of microwave communication through the fat tissue. The muscle tissue was considered as a perturbant and is placed inside the fat tissue to investigate the performance of the channel. Perturbant sizes in the range from $5 \mathrm{~mm}$ cube to $20 \mathrm{~mm}$ cube are used in this study. Two perturbant sizes were selected from the simulation and used in the phantom measurements. The results show that the signal coupling through the fat channel degrades when a perturbant of $15 \mathrm{~mm}$ cubes or more is introduced into the channel. The results indicate that the fat channel has substantial resilience (up to $60 \%$ channel height) towards perturbing scenarios such as the presence of an embedded muscle layer and blood vessels. This work is an initial study towards understanding the reliability of the fat tissue when used as an intra-body communication channel.

\section{ACKNOWLEDGMENT}

This work was supported by the Ministry of Higher Education, Malaysia, a Eurostars project (grant E-9655COMFORT), Swedish Vinnova projects, BDAS (2015-04159) and Reliable, interoperable and secure communication for body network (2017-03568) and a strategic collaborative eScience program by the Swedish Research Council (grant eSSENCE).

\section{REFERENCES}

[1] M. S. Wegmüller, "Intra-body communication for biomedical sensor networks," Ph.D dissertation, ETH Zurich, 2007.

[2] Y. Terauchi, et al., "Analytical and Experimental Modeling Of IntraBody Communication Circuit," in Mechatronics for Safety, Security and Dependability in a New Era, edited by Eiji Arai and Tatsuo Arai, Elsevier, Oxford, 2007, pp. 409-414.

[3] I. F. Akyildiz, M. Pierobon, S. Balasubramaniam and Y. Koucheryavy, "The internet of Bio-Nano things," in IEEE Communications Magazine, vol. 53, no. 3, pp. 32-40, Mar. 2015.

[4] M. Seyedi, B. Kibret, D. T. H. Lai and M. Faulkner, "A Survey on Intrabody Communications for Body Area Network Applications," in IEEE Transactions on Biomedical Engineering, vol. 60, no. 8, pp. 2067-2079, Aug. 2013.

[5] N. B. Asan et al., "Intra-body microwave communication through adipose tissue", Healthcare Technology Letters, 2017, vol. 4, no. 4, pp. $115-121$.

[6] N. B. Asan et al., "Human fat tissue: A microwave communication channel," 2017 First IEEE MTT-S International Microwave Bio Conference (IMBIOC), Gothenburg, 2017, pp. 1-4.

[7] Asan, Noor Badariah et al., "Data Packet Transmission through Fat Tissue for Wireless Intra-Body Networks" submitted.

[8] You and your hormones, Society for Endocrinology. Available: http://www.yourhormones.info/glands/adipose-tissue/, accessed August 2017.

[9] CST - Computer Simulation Technology. Providing complete technology for high frequency 3D EM field simulation. Available: https://www.cst.com/products/cstmws/solvers, accessed July 2017.

[10] S. Gabriel, R. W. Lau, C. Gabriel, "The dielectric properties of biological tissues: II. Measurements in the frequency range $10 \mathrm{~Hz}$ to 20 GHz," Physics in Medicine \& Biology, vol. 41, pp. 225-2269, 1996.

[11] E. Hassan, D. Noreland, E. Wadbro and M. Berggren "Topology Optimisation of Wideband Coaxial-to-Waveguide Transitions," Scientific Reports 7, 45110, Mar. 2017. doi: 10.1038/srep45110.

[12] Keysight Techologies. N9918A fieldfox handheld microwave analyzer, 26.5 GHz. Available: http://www.keysight.com/en/pdx-x201927-pnN9918A/fieldfox-handheld-microwave-analyzer-265-ghz?nid=32495.1150498\&cc=SE\&lc=eng, accessed July 2017.

[13] Keysight Techologies. 8507E Dielectric probe kit. Available: http://www.keysight.com/en/pd-304506-pn-85070E/dielectric-probekit?cc=SE\&lc=eng, accessed July 2017. 